\section{Reconocer lo político como una esfera propia del ser humano en la educación virtual}

Recognizing the political matter

as a proper sphere of the human being in virtual education

\section{Reconhecimento da política como espaço próprio do ser humano na educação virtual}

\section{Diana Paola Sáenz Castro*}

Magister en Educación, de la Universidad Pedagógica Nacional Docente de la Fundación Universitaria Los Libertadores y de la Alianza Universidad Tecnológica de Bolívar-EDUPOL.

Correo electrónico: dsaenzcastro@gmail.com

\section{Resumen}

Bajo la premisa de que reconocer lo político como una esfera propia del ser humano y de la sociedad permite direccionar tendencias y prioridades en términos de formación, el presente artículo de reflexión parte de dos preguntas centrales: ¿cuáles son las políticas subyacentes a la educación virtual?, ¿qué tendencias políticas está aportando lo virtual a la educación?

\section{Palabras clave}

Educación virtual, política, sujetos, redes.

\section{Abstract}

Under the premise of recognizing the political matter as proper sphere of the human being and the society permits to direct tendencies and priorities in terms of education, this article of reflection starts from two central questions: What are the underlying policies to virtual education?, What political tendencies is the virtual matter contributing to education?

\section{Key words}

Virtual education, policy, individuals, networks.

\section{Resumo}

Baseado em que reconhecer que a política pertence à esfera do ser humano e da sociedade permite orientar tendências e prioridades em quanto formação, este artigo de reflexão se fundamenta em duas questões importantes: que políticas subjacem à educação virtual ? e que tendências políticas fornece o virtual à educação?

\section{Palavras-chave}

Educação virtual, política, redes, sujeitos.

Fecha de recepción: 21 de septiembre de 2009 Fecha de aprobación: 17 de febrero de 2010

\section{Fecha de aprobación: 17 de febrero de 2010}




\section{El concepto de lo político, la cultura política democrática y las redes como un ejercicio político}

$\mathrm{D}$ efinir el concepto de lo político implica, ante todo, reconocer su complejidad semántica. Si nos remitimos a su origen en el idioma inglés es posible reconocer tres acepciones que le dan sentido al concepto: "Primero, la política concebida como el ámbito del gobierno de las sociedades humanas; polity, en inglés. Segundo, la política como la actividad de organización y la lucha por el control del poder; politics, en inglés. Y, finalmente, la política como designación de los propósitos y programas de las autoridades públicas; policy, en inglés" (Roth, 2002, p. 5).

En los escenarios políticos, se lucha por el posicionamiento de una ideología política sobre otras. El campo de la Educación virtual no escapa de esta fuerte tensión, referida a la organización y lucha por el control del poder; por el contrario, parece contribuir a escenificar determinadas ideologías y a posicionar unas por encima de otras. De igual manera, hace evidente que las políticas locales, en este caso las educativas, dependen de las políticas internacionales.

En este último sentido, las políticas nacionales se convierten en espacios de interacción de distintos intereses. Por un lado, quienes defienden que la función del Estado en materia de política educativa consiste en satisfacer la demanda educativa del mercado (Suárez, 2007); en consecuencia, se ha de organizar en torno a la política internacional, pues la educación se considera como elemento decisivo para lograr la transición de un modelo económico a otro. De otra parte, quienes consideran que la acción educativa del Estado puede contribuir a la transformación de la sociedad en favor de la mayoría de los ciudadanos. Ahora bien, sin considerar la política educativa colombiana de manera aislada de la internacional, de gobierno de turno y de las políticas de inversión (Suárez, 2007), puede responder a esta transformación.

Los fenómenos sociales, políticos, culturales y económicos asociados con la globalización y con el neoliberalismo, como tendencias mundiales, terminan por afectar las políticas educativas en Colombia. De ahí que sea necesario referirnos al surgimiento de la globalización que influye en las esferas del ser humano y al modo cómo este fenómeno atraviesa los discursos relacionados con la política, la educación y, de manera particular, con la educación virtual. Al respecto, Pérez Gómez (2004) considera que en la actualidad nos vemos abocados a procesos de globalización de los intercambios económicos-por la flexibilidad y fluidez de los procesos productivos, de distribución y consumo-. Este acontecimiento plantea para los ciudadanos nuevos desafíos, pero también nuevas incertidumbres por la rapidez y la extensión de los cambios que se suscitan en los más amplios y diversos aspectos de la vida.

De la misma manera, nos enfrentamos a la confusión, a la fragmentación y a la perplejidad que hoy día sustituyen las costumbres del pasado y que ya no de hacen suficientes para afrontar los nuevos desafíos y las exigencias del tiempo presente. Tal insuficiencia ha provocado que los cambios profundos de la sociedad contemporánea hayan ido convirtiendo en obsoletos los modelos tradicionales de la escuela $y$, de igual manera, pareciera que no existen claras orientaciones respecto de los nuevos rumbos de la educación. Como lo señala Díaz Barriga (2003), se niega la posibilidad de mejorar los procesos educativos, la formación de los licenciados, la integración y la vinculación del contenido curricular con la realidad social, política y científico-tecnológica. Trasmite el ideal de generar mejores escuelas, mediante la búsqueda de los mejores docentes y estudiantes $\mathrm{y}$ al relegar del medio educativo a estudiantes con necesidades especiales con el argumento de que son costosos y desacreditan a las instituciones.

De otra parte, si se asume la globalización, desde la visión de Pérez Gómez (2004), como un fenómeno esencial de la economía neoliberal, observamos igualmente una fuerte tendencia de esta misma economía a la imposición universal de modelos de vida, de pensamientos, de sentimientos y conductas que se transmiten a través de los medios de comunicación de masas. Imposición que contribuye a la formación de una ideología social en la cual, sin una postura crítica, todo vale

“En los escenarios políticos, se lucha por el posicionamiento de una ideología política sobre otras. El campo de la Educación virtual no escapa de esta fuerte tensión, referida a la organización y lucha por el control del poder; por el contrario, parece contribuir a escenificar determinadas ideologías y a posicionar unas por encima de otras." 
tanto moral como política y socialmente en cuanto sirve al fin primordial de la rentabilidad personal, grupal y nacional.

El fenómeno de la globalización se encuentra íntimamente relacionado con el del neoliberalismo, según lo planteado por Apple (2001). En este escenario, el Estado actúa como un ente que legisla y vigila a sus ciudadanos. Y en lo educativo, por su parte, el Neoliberalismo instaura indicadores de desempeño estandarizados.

Es posible pensar que la Educación Virtual se halla en la encrucijada de responder a los retos planteados por diversos modelos socioeconómicos y políticos, según la condición de responder a indicadores internacionales, pero, al mismo tiempo, a ideologías de transformación social que deben respetar las características propias de los contextos, con el objeto de romper un poco con la idea de homogeneización de los grupos. La transformación de la educación, y de modo específico, de la educación virtual no ha de partir desde los grandes modelos sociales, políticos y educativos. La educación virtual que hace un aporte a las transformaciones sociales requeridas, ha de gestarse en el marco de los procesos de formación y de construcción de una sociedad democrática. Igualmente, los procesos investigativos pueden contribuir a consolidar argumentos en favor de la educación en entornos digitales; es decir, contribuir a la reflexión acerca del uso de entornos digitales en favor de la formación del ser humano. En última instancia, el reto se da desde los procesos que se gestan en torno a la educación virtual: asumir una posición autónoma, crítica y reflexiva; en otras palabras, asumir un rol y un posicionamiento político, como un primer paso, para

“...la Educación Virtual se halla en la encrucijada de responder a los retos planteados por diversos modelos socioeconómicos y políticos, según la condición de responder a indicadores internacionales, pero, al mismo tiempo, a ideologías de transformación social que deben respetar las características propias de los contextos, con el objeto de romper un poco con la idea de homogeneización de los grupos." avanzar en la formulación y en el mejoramiento de políticas educativas concretas que vayan más allá de requerimientos técnicos respaldando procesos democráticos, formativos, investigativos y de desarrollo profesional.

Por lo anterior, para el caso de la Educación virtual, y para la caracterización del contexto colombiano, se hace particularmente relevante referirnos a la política como polity. Se habla, en consecuencia, de la política como el ámbito del gobierno de las sociedades humanas $y$, por ende, desde este momento se le considerará no sólo desde los requerimientos técnicos sino también desde los pilares de la cultura política democrática.

\section{La cultura política democrática}

Jacqueline Peschard (2001) define la cultura como un conjunto de símbolos, normas, creencias, ideales, costumbres, mitos y rituales que se transmite de generación en generación, que otorga identidad a los miembros de una comunidad y da consistencia a la sociedad que condensa herencias, imágenes y experiencias colectivas con las cuales se da a la población sentido de pertenencia. La política se define como el ámbito de la sociedad relativo a la organización del poder; se reconoce como el espacio donde se adoptan decisiones que inciden en la proyección social y se define la distribución de los bienes de una sociedad.

Por su parte, la cultura política, se define como el conjunto de valores, concepciones y actitudes orientadoras de lo político; es decir, el conjunto de elementos que configuran la percepción subjetiva que tiene una población respecto del poder. El concepto nació con la modernización, con la transición de una sociedad tradicional a una moderna, caracterizada por la introducción de de la tecnología al proceso productivo y por el desafío de poder lograr nuevas estructuras políticas y nuevas relaciones de poder que dinamizan las demandas de los grupos sociales (Peschard, 2001).

Esa cultura política, que responde a las nuevas demandas de los actores sociales, consensúa valores y normas a partir de principios democráticos y respeta el sentido de ciudadanía como cualidad del sujeto protagonista de la esfera pública. El sujeto, entonces, se convierte en participante directo o indirecto en la fundamentación del poder del Estado y en la idea de una sociedad atenta y vigilante.

De igual manera, el sujeto se reconoce como actor de una sociedad abierta -activa y deliberativa con amplios márgenes de autonomía-, de la 
secularización -que rompe barreras impuestos por dogmas religiosos-, de la competencia -para estar convencidos de que se puede hacer algo- de la legalidad-que define sanciones frente a conductas que violan normas aplicadas a todos por igual- y de la pluralidad-que permite coexistir en el mismo espacio político, al respetar la diversidad-. Aspectos que no serían posibles si no se tomara el hecho de asumir la cultura política desde la cooperación como asunto realmente importante.

\section{Las redes como un ejercicio político}

En la época de cambio de siglo se ha venido evidenciando la creación y el uso de las redes electrónicas como base del surgimiento de un nuevo tipo de sociedad que se construye y se desarrolla en cualquier espacio que cuente con los medios para comunicarse. Época de políticas de transformación social y de nuevos actores políticos, como respuesta a cambios significativos en la manera de abordar las preguntas y definir las acciones que se derivan de la búsqueda de experiencias prácticas. Gracias a las redes, el conocimiento de las

"Dado que las redes producen una particular forma de mirar el mundo, no tanto en términos de fragmentación sino de posibilidad de coalición, se hace necesario enunciar el uso de tales redes como una estrategia de reconocimiento de la diversidad."

\section{Metodología de la revisión documental}

Luego de enunciar las tendencias globalizadoras y neoliberales que enmarcan el concepto de política, la cultura política democrática, las cualidades del sujeto como actor que participa continuamente en la transformación de la sociedad y cómo las redes se han constituido en motores de construcción de conocimiento a partir de las experiencias, se describe en adelante cómo se ha entendido la educación virtual y qué se encuentra acerca de políticas en el campo a partir de la revisión documental guiada por las preguntas eje de la investigación: ¿Cuáles son las políticas subyacentes a la educación virtual? ¿Qué tendencias políticas está aportando lo virtual a la educación?

Para dar respuesta a estos interrogantes, la búsqueda se centró en diversos documentos que circulan en la web y que están relacionados con los significados, los usos y las concepciones que se le atribuyen a la educación virtual y, también, con las tendencias políticas evidenciadas en tales concepciones, significados y usos. Especialmente se indagaron los portales educativos de España, Chile, México y Colombia y las investigaciones publicadas en revistas referidas por las páginas Pixel-bit, Edutec y Redalyc, en el lapso comprendido entre los años 2003 y 2008.

\section{Las concepciones de educación virtual}

De manera específica, las concepciones de educación virtual se construyen a partir de los usos y las cualidades derivadas de la necesidad de crear redes de conocimiento y de responder a lineamientos de política internacional enmarcados por la globalización y el neoliberalismo. Sin embargo, lo consultado parece confluir al mismo punto: no existen relaciones claras entre lo esperado de la educación virtual y de la mediación pedagógica a partir de tecnologías de información y lo que realmente se obtiene; por tanto, no existen políticas definidas. una característica de las redes que se basa en una noción positiva de la diferencia. 
Al parecer, existen diversos y variados conceptos acerca del tema requieren que requieren profundización y análisis, por cuanto esta falta de claridad impide avanzar en la reconstrucción de estrategias de aprendizaje y en la construcción de conocimiento evidenciado en propuestas de desarrollo sostenibles que transformen el entorno. Castañeda (2007) define la educación virtual desde un entorno de enseñanza-aprendizaje en el cual los modelos de tutoría, debate o simple diálogo se abren al educador con un gran catálogo de opciones para ser usados en casi cualquier momento y lugar y con diversas repercusiones en el aula que cada vez tiene menos necesidad de estar delimitada por paredes. Opciones y usos que se configuran en tanto que el entorno de aprendizaje personalizado se hace posible a partir de las herramientas que configuran la visión particular que cada quien tiene acerca de su entorno para aprender, bien sea leyendo, conversando, compartiendo o simplemente "fisgoneando" (lurking) ${ }^{1}$.

La Educación Virtual es entendida también como el entorno de aprendizaje, concebido por Dewey como el espacio donde los alumnos pueden interactuar y estudiar de qué manera aprender (Dewey en Joyce y Weil, 2002) o concebido como el espacio o comunidad organizados con el fin de lograr el aprendizaje (Jesús Salinas, 2004); en otras palabras, la Educación Virtual es un espacio que facilita la interacción entre los actores que se relacionan en el proceso educativo. Si al anterior concepto (entorno de aprendizaje) se le suma la ayuda de TIC, se puede definir como el conjunto de recursos concretos, hardware y software utilizados en la enseñanza como sucede en las aulas de ordenadores con acceso a Internet; se le considera entonces una metáfora de un lugar de estudio (Wilson, 1996). Van Dusen (1997) también define el entorno de aprendizaje con ayuda de TIC, como una metáfora de la enseñanza

1 El propósito del trabajo de Castañeda consiste en explorar algunos de los elementos tecnológicos emergentes en el mundo de la llamada Web 2.0 y que pretenden ahondar en conceptos como long-life-learning y la integración de los procesos de enseñanza formal, no formal e informal en procesos globales que desdibujan las paredes de las instituciones educativas tradicionales hacia modelos más colaborativos, abiertos y de construcción compartida. La metodología empleada parte de las tres metáforas de implementación de las herramientas telemáticas planteadas por Adell: las redes como biblioteca, como imprenta y como canal de comunicación (Adell, 2004). Analizamos algunas de las nuevas herramientas que han hecho su aparición en la red de redes en los últimos tiempos en un intento por hacer no sólo una descripción de sus características sino también un examen de las posibilidades educativas que nos plantean dichas herramientas en los procesos de enseñanza formal. electrónica, en el que el ambiente de aprendizaje es creado por una tecnología de la información.

La Fundación para el Desarrollo del Conocimiento FUNDESCO (1998) introduce el término virtual al entorno de aprendizaje y lo define como el programa informático interactivo de carácter pedagógico que posee una capacidad de comunicación integrada, asociada a nuevas tecnologías. McCormack y Jones (1998) se refieren al entorno de aprendizaje desde la expresión Webbased classroom; un entorno creado en la Web en el cual estudiantes y educadores pueden llevar a cabo tareas de aprendizaje. Khan (2001) utiliza los términos Web-based Training (WBT) y Web-based Instruction (WBI) para referirse a programas de instrucción basados en los hipermedia y dirigidos a una audiencia remota, utilizando los atributos y recursos de la web para crear ambientes de aprendizaje bien diseñados, centrados en los alumnos, interactivos y facilitadores².

\section{Lo virtual en educación}

Se enuncian aquí algunas concepciones de lo virtual en educación halladas en la revisión de artículos de investigación acerca del tema y publicados en redalyc.uaemex.mx entre 2003 y 2008.

Lo virtual como comunidad. Ocampo (2008) considera lo virtual como el entramado de relaciones y actividades que constituyen un mundo electrónico que reta los referentes habituales; el espacio virtual se entiende como un espacio que satisface necesidades y como un espacio de interacción con personas que posibilita las comunidades sociales, de manera solidaria, al buscar la participación y el compromiso de los demás (Parra, 2008).

Lo virtual como ruptura de la monotonía. Lo virtual cuenta con actividades que desplazan los referentes habituales de la vida cotidiana (Ocampo, 2008); posibilitan los vínculos con comunidades a las que sería complejo tener acceso (Parra, 2008). Lo virtual ofrece un contexto y un proceso de comunicación diferente al impreso y a la presencialidad (Torres, 2005).

Lo virtual como un mundo paralelo. Espacio que ofrece la posibilidad de participar sin los prejuicios de lo físico (Ocampo, 2008) o con mayor comodidad (Parra, 2008).

2 Las definiciones enunciadas hacen parte del artículo "Los entornos tecnológicos en la universidad", cuyo propósito radica en describir las principales características que presentan las herramientas susceptibles de utilizarse en entornos virtuales de formación. 
de contacto entre maestros que implementan TIC en beneficio de los planes educativos creados por áreas comunes. Incluye el sentido de la innovación como necesidad marcada por políticas económicas mundiales así como el enfoque de competencias que, como producto, genera una propuesta docente que orienta el uso de TIC y que evidencia claramente el desarrollo de la autorregulación en el aprendizaje y de la autonomía por parte de quien, a partir del estudio de casos, aprende y logra como resultado el aprendizaje cooperativo. Para lograr este objetivo, se hace necesaria una planificación en grupo en relación con las metodologías de enseñanza, seguimiento y evaluación al desarrollo del aprendizaje de los estudiantes mediado por entornos digitales (Mauri, T., 2009).

Políticas de información. Almada de Ascencio (1999, p. 34) concibe las políticas de información como "el estudio de las orientaciones y directrices que rigen la actuación de los diferentes sectores o grupos de la sociedad en la transferencia de información, su disponibilidad, su acceso y recuperación como medio para la comunicación de información entre personas generadoras y usuarios de información". Esta definición ha sido usada dentro del campo disciplinar de la bibliotecología para entender las acciones sociales, proveer datos y documentos y tejer alianzas con pares, editores y proveedores de tecnologías de la información (Sánchez, J., 2006).

La formación online. Esta investigación centrada en la evaluación pretende indagar por la forma cómo los docentes aplican recursos de las plataformas educativas en el desarrollo de sus cursos (Ramírez, 2006). Para lo anterior, es necesario haber formado los maestros en el tema y brindarles diversas opciones que contribuyan a su desarrollo profesional como maestros, al asumir la formación desde las competencias docentes de índole instrumental, sistémica y aplicada y pensar en el impacto generado en los estudiantes como actores de la formación disciplinar. Los resultados de la evaluación sirven para establecer orientaciones pedagógicas para la formación del profesorado (Colás, p. 2007).

Políticas y formas de incorporación de las TIC en algunos países de Latinoamérica. Los gobiernos de la región han encaminado esfuerzos hacia la construcción de sociedades de la información en sus países y han obtenido sumas de dinero para consolidar estas iniciativas. Por este motivo, en los países latinoamericanos se han conformado redes de información, redes de escuelas y redes de educación a distancia que, de manera infortunada, parecen desconocer una adecuada contextualización cultural y socioeconómica y, de este modo, se aleja la posibilidad de enmarcar las propuestas como cualificadas en la autosustentabilidad. El tema de la incorporación de tecnologías de la información y la comunicación se viene desarrollando de diversas maneras. Pueden observarse experiencias piloto, iniciativas particulares o propuestas de organismos internacionales. También son ya conocidas las posturas en Latinoamérica que apuntan a limitarse a conectividad o compra de equipos.

Las tecnologías de la información y de la comunicación en la educación en cuatro países latinoamericanos. El artículo expone cómo se ha desarrollado el tema de la incorporación de TIC en Ecuador, Costa Rica, Argentina y México. Para los académicos ecuatorianos, la falta de políticas claras o debidamente coordinadas, limita la visión nacional acerca de los alcances de la educación virtual. Los lineamientos que alcanzan a referirse al tema, son producto del interés de investigadores y docentes en educación superior. Al parecer, las políticas de incorporación en Ecuador se evidencian en los incentivos económicos dados a los docentes que se forman en el tema y a aquellos que dan uso a las tecnologías de alguna forma. La incorporación de centros comunitarios en zonas rurales puede ser tan limitada como la capacitación a docentes en el área de informática básica.

En Costa Rica, se ha llevado a cabo la construcción de laboratorios de informática educativa para escuelas urbanas grandes y pequeñas redes de computadores para planteles rurales con el objeto de prever servicios y herramientas de telecomunicaciones a las escuelas. Para los docentes costarricenses la instalación de laboratorios de informática posibilita su capacitación en el área. Microsoft también ha hecho aportes a los docentes al capacitarlos en el aprovechamiento de Microsoft Office e integrando, además, el uso de los computadores en el plan de estudios; se pretende con esto mejorar el aprendizaje y el desempeño de los estudiantes.

Argentina no cuenta con un plan nacional o provincial, a pesar de las buenas iniciativas, proyectos y redes llevadas a cabo en diferentes instituciones. Cuenta, como los países enunciados anteriormente, con campañas para incorporar computadores en las escuelas públicas, para acceder a equipos y para conformar redes. La diferencia notoria entre Argentina y otros países la marca el hecho de pensar en el acercamiento de las minorías: cuenta con programas que buscan acercar a personas con necesidades educativas especiales, a enfermos crónicos y a ancianos. El proyecto Nueva Alejandría 
acompaña a los docentes en la incorporación de TIC en los procesos de enseñanza aprendizaje.

México se concentra en proyectos que usan de manera integral las tecnologías; organizan redes de carácter comunitario a partir de proyectos colaborativos.

Aunque existen intereses de organizaciones internacionales como la Fundación Ford, no hay lineamientos pedagógicos claros que lleguen más allá de la necesidad de usar tecnologías. Sin embargo, se han diseñado aplicaciones para la enseñanza de materias básicas de educación secundaria. La Universidad Técnica Particular de Loja en Ecuador es pionera en educación a distancia, ejemplo más preciso en la aplicación de las TIC en el ámbito universitario. La Universidad de Quilmes en Argentina, cuenta con el primer campus virtual. El Instituto Tecnológico de Estudios Superiores de Monterrey, a través del proyecto de Universidad Virtual, cuenta con ofertas de Licenciatura, Maestría y Doctorado; además, del programa de actualización en habilidades docentes (Ramírez, J., 2006).

Factores que orientan la calidad en la Educación virtual. La investigación llevada a cabo por Light (2005) con el apoyo de REDAL (Redes Escolares de América Latina) ha permitido identificar factores clave que promueven una inserción exitosa de TIC en la educación a través de redes escolares. A partir de la identificación de factores, se pretende generar lineamientos como guía para formular políticas educativas en lo referente a TIC y a redes escolares en Latinoamérica. Los factores claves identificados son:

- Los objetivos: incluyen la misión de la red y el propósito pedagógico.

- El liderazgo: incluye la formación en liderazgo (el líder crea capacidad de liderazgo en los demás, comunica los objetivos de la red, tiene perspectiva de cambios a largo plazo, reconocen la extensión y profundidad del problema).

- El desarrollo profesional: tanto a nivel técnico como pedagógico, de forma presencial o en línea.

- Experimentación, reflexión sobre la práctica y adaptación: comenzar con proyectos pilotos.

- Tiempo: para lograry gestionar implementación de la red.

- Infraestructura: incluye el equipamiento (relación entre equipos disponibles y actividades propuestas y el mantenimiento y actualización), la conectividad (vela por la calidad de la conexión y las políticas de telecomunicaciones) y el ac- ceso a equipos y recursos. El Financiamiento y sustentabilidad, la pertenencia, la comunicación significativa a través de las TIC, el propósito común, relacionado con la Misión de la red y el protagonismo y liderazgo de participantes con objetivos comunes, también son considerados por esta investigación como factores clave.

La formación del profesorado en TIC. El estudio de las políticas en la formación del profesorado europeo en TIC evidencian altos niveles de desarrollo en competencias instrumentales, sistémicas y aplicadas que inciden en la integración efectiva dentro de las estructuras curriculares, así como la activación de acciones formativas para los maestros que se concentran en qué hacen en los contextos escolares con las TIC para cambiar el currículo y los aprendizajes. Ello obedece a que se cree que los beneficios de las tecnologías en los centros escolares y en las aulas son sólo posibles a través de una idónea contextualización de las mismas (Colás, 2008).

\section{Normatividad colombiana acerca de la Educación Virtual en la Educación Superior}

Con el fin de complementar la descripción de las tendencias políticas que orientan la educación virtual en nuestro contexto, y con el objetivo de hacer una aproximación a las concepciones, usos y tendencias de la misma en la política educativa de educación superior, se presenta a continuación una síntesis de lo hallado. Información que, aun cuando se relaciona con la educación virtual, no la define.

El Decreto 2566 de Septiembre 10 de 2003 -por el cual se establecen las condiciones mínimas de calidad y demás requisitos para el ofrecimiento y desarrollo de programas académicos de educación superior y se dictan otras disposiciones- aborda aspectos como los medios educativos cuando en parágrafo de su décimo artículo determina que:

Para programas que se desarrollen en la metodología de educación a distancia, la institución deberá disponer de los recursos y estrategias propios de dicha metodología, a través de las cuales se atiende el acceso permanente de todos los estudiantes y profesores a la información, experimentación y práctica profesional, necesarias para adelantar procesos de formación, investigación y proyección social. Igualmente, se demostrará la existencia de procedimientos y mecanismos empleados para la creación, producción, distribución y evaluación de materiales de estudio, apoyos didácticos y recursos tecnológicos con soporte digital y de telecomu-

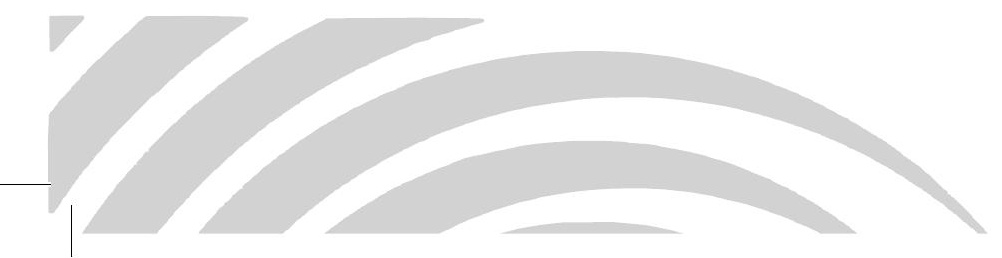


nicaciones, y acceso a espacios para las prácticas requeridas.

Las condiciones mínimas de calidad se refieren a requerimientos técnicos que fortalecen los procesos de formación, investigación y proyección social, por medio de la creación de materiales de estudio. El artículo número once, referido a la infraestructura, se suma a los requerimientos técnicos, resultado de las demandas del mercado enunciadas anteriormente. Determina, además, que los programas desarrollados desde la metodología a distancia deben ubicar equipos e inmuebles en los lugares ofrecidos.

La Resolución 2755 de 2006 -por la cual se definen las características específicas de calidad para la oferta y desarrollo de los programas académicos en la metodología a distancia- aborda, al igual que el Decreto 2566, el aspecto de los medios educativos y sugiere, en su $5^{\circ}$ artículo, el uso de laboratorios virtuales, de apoyo a la actividad académica. El primer artículo de la Resolución define, asimismo, la educación superior a distancia como aquella metodología educativa que se caracteriza por utilizar ambientes de aprendizaje en los cuales se hace uso intensivo de diversos medios de información y comunicación y de mediaciones pedagógicas que permiten crear una dinámica de interacciones orientada al aprendizaje autónomo y abierto; superar la docencia por exposición y el aprendizaje por recepción, así como las barreras espacio-temporales y las limitaciones de la realidad objetiva mediante simulaciones virtuales; adelantar relaciones reales o mediadas y facilitar aprendizajes por indagación y mediante la colaboración de diversos agentes educativos. Se considera pertinente resaltar en esta Resolución la pretensión de simulaciones virtuales para superar las limitaciones de la realidad objetiva.

\section{Hallazgos generales en cuanto a las tendencias políticas en Educación Virtual y la transformación social}

La globalización y el neoliberalismo como modelo económico que desconoce contextos específicos. Por un lado, la política como fundamento de la educación y de sus acciones ha sufrido vertiginosas transformaciones en el siglo XX y XXI. Con una enorme rapidez se han constituido dos grandes fenómenos socio-políticos y económicos que parecen imperar en el escenario mundial: la globalización y el neoliberalismo. Ambas de origen económico pero que terminan por acceder a todos los contextos de la vida humana.
La globalización se caracteriza porque desde la base del libre intercambio de bienes y servicios, y con el apoyo permanente de los medios masivos de comunicación, termina por universalizar diversos modelos de vida, lo cual genera incertidumbre entre las personas y relativiza valores y formas de ser y hacer.

Por su parte, el neoliberalismo supedita todas las esferas del ser humano individual y colectivo a las reglas del mercado, a la competitividad, al fomento de los mejores y la supresión de los peores. En el campo de la educación, el neoliberalismo pone en marcha competencias de estudiantes, establece clasificaciones, otorga incentivos a los primeros y relega a los últimos. En última instancia, se asume a la educación y a la escuela como una gran empresa.

La cultura política democrática y el trabajo en red. El tema de cultura política democrática, y cada uno de sus componentes, permite pensar la posibilidad de un grupo de alternativas que, fundamentadas en la investigación, establecezcan y legitimenpolíticas en torno a la educación virtual; políticas que vayan más allá de la implementación de TIC en la educación, impuestas por órdenes políticos internacionales y que piensen en los grupos sociales como gestores de transformación en beneficio de la sociedad. Su herramienta, la creación de redes, como una estrategia de reconocimiento de la diversidad.

La educación virtual y la formación en competencias. El enfoque de la formación en competencias que responde a la ideología neoliberal, pero que va más allá del discurso de formación de mano de obra barata, se evidencia en la autorregulación en el aprendizaje y en la autonomía, ejercicios que tienen un vasto campo en la educación virtual y sobre los cuales se han construido algunas propuestas enunciadas anteriormente, relacionadas con habilidades cognitivas y con procesos de interacción.

El tema de las competencias en educación virtual no ha sido pensado sólo para los procesos de aprendizaje de los estudiantes; también para los procesos de enseñanza de los maestros. Al formar a un maestro en el uso de nuevas tecnologías, se le brindan diversas opciones en beneficio de su desarrollo profesional, pensando en el impacto generado en los estudiantes como actores de la formación disciplinar. El estudio de políticas de formación en TIC del profesorado europeo, enunciado en páginas anteriores, evidencia un alto nivel de desarrollo en competencias instrumentales, sistémicas y aplicadas que inciden en la interdisciplinariedad curricular e inciden, además, en los programas de 
formación docente que se basan en el reconocimiento de los contextos para atender necesidades específicas de los mismos.

El uso de TIC, la innovación y la mejora de procesos de enseñanza y aprendizaje. El uso de TIC rompe barreras espacio-temporales en los procesos de enseñanza y aprendizaje; la información que hace unos años tardaba días en llegar de una región a otra, de un país a otro, ahora se puede conseguir de manera casi inmediata. La incorporación de TIC a las universidades dista mucho aun de ofrecer programas curriculares apoyados en su totalidad por el uso de tecnologías, pero no se puede negar que se ha logrado una mayor aproximación de estudiantes y maestros al conocimiento. Las metodologías presenciales y los entornos digitales inciden en una forma diferente de acciones que se desarrollan en el ser humano que dependen de la constancia y la pertinencia con que se utilizan.

Lo virtual como comunidad, como ruptura de la monotonía, como mundo paralelo. Se considera lo virtual como un entramado de relaciones que, al posibilitar la creación de comunidades, satisface necesidades y permite la interacción. En tanto que ofrece un contexto diferente a la presencialidad, ha venido desplazando referentes habituales de la vida cotidiana. Además, ofrece la posibilidad de participar con mayor comodidad.

\section{Bibliografía}

Apple, M. (2001), “¿Pueden las pedagogías críticas interrumpir las políticas neoliberales?”, en Opciones Pedagógicas, núm. 24, Bogotá, Universidad Distrital Francisco José de Caldas, Facultad de Ciencias y Educación.

Díaz Barriga, A. (2003), Mesa de trabajo 1, evaluar lo académico. Organismos internacionales, nuevas reglas y desafíos, Bogotá, Universidad Pedagógica Nacional, encuentro regional sobre políticas en evaluación educativa.

Pérez Gómez, Á. (2004), “La construcción del sujeto en la era global”, en: Encuentro Internacional sobre políticas, investigaciones y experiencias en evaluación educativa. Consecuencias para la educación, Bogotá, Universidad Pedagógica Nacional, Facultad de Educación.

Peschard, J. (2001), La cultura política democrática, México, Instituto Federal Electoral.
Hacia una alternativa política desde la educación virtual. Concebir la política como el ámbito del gobierno de las sociedades humanas, permite hacer a un lado la tendencia individualista de la autonomía; esta tendencia, impide el trabajo en comunidad por el logro de un objetivo común. Como respuesta al neoliberalismo y a la globalización, surgen fenómenos desde lo social que van en contra de la imposición universal de modelos de vida, pensamientos, sentimientos y conductas. Es posible pensar que la educación virtual se halla en la encrucijada de responder a los retos planteados por diversos modelos socioeconómicos y políticos; en especial al tener en cuenta que se debe responder a indicadores internacionales, pero al mismo tiempo a ideologías de transformación social. De ahí que la educación virtual ha de gestarse en el marco de los procesos de formación y de construcción de una sociedad democrática, que haga evidente de forma clara el desarrollo de la autorregulación en el aprendizaje y la autonomía por parte de quien aprende al lograr, como resultado, el aprendizaje cooperativo. Sin embargo, esto es poco probable si quien enseña no ha sido formado en el tema de la educación virtual, del trabajo en redes o del aprendizaje autónomo, entre otros, y si no ha iniciado procesos de investigación que permitan la transformación de una enseñanza presencial a los nuevos entornos digitales.
Roth, A. (2002), “Conceptos, teorías y herramientas para el análisis de las políticas públicas", en Políticas públicas. Formulación, implementación y evaluación, Bogotá, Aurora. Citado en Observatorio Nacional de Políticas en Evaluación (2005). “El Observatorio Nacional de Políticas en Evaluación, una propuesta de la Universidad Pedagógica Nacional", en Serie documentos para una evaluación crítica, núm. 0 , Bogotá, Universidad Pedagógica Nacional.

Suárez, P. A. (2007), Incidencia de la actual política evaluativa en instituciones de educación básica del país. Observatorio Nacional de Políticas en Evaluación, Bogotá, Universidad Pedagógica Nacional.

Barroso, C. (2006), "Elementos para el diseño de entornos educativos virtuales con base en el desarrollo de habilidades", en Edutec. Revista Electrónica de Tecnología Educativa. 2, [en línea], 1, disponible en: http:// edutec.rediris.es/Revelec2/revelec21/cbarrosol. htm, recuperado: Agosto de 2009.

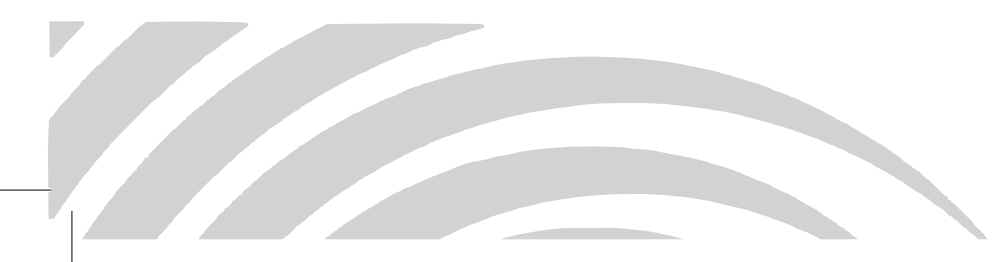


Castañeda Quintero, L. (2007), "Software social para la escuela 2.0: más allá de los Blogs y las Wikis" En Inclusión Digital en la Educación Superior: Desafíos y oportunidades en la sociedad de la Información. X Congreso Internacional EDUTEC 2007, [en línea], Buenos Aires: Universidad Tecnológica Nacional, Disponible en: http://www.lindacastaneda.com/ publicaciones/edutec20071.pdf, recuperado: Agosto de 2009.

Castañeda, L. (2009), “Las universidades apostando por las TIC: modelos y paradojas de cambio institucional”, en EDUTEC-E Revista electrónica de tecnología Educativa, [en línea], disponible en: http://edutec. rediris.es/Revelec2/revelec28/edutec28_universidades_apostando_tic.html, recuperado: Mayo de 2009.

Colás, P. (2008), "Evaluación del impacto de la formación (online) en TIC en el profesorado. Una perspectiva sociocultural", en Revista de Educación, [en línea], 346, pp. 187-215, disponible en: http://www.revistaeducacion.mec.es/re346/re346_07.pdf, recuperado: Junio de 2009.

Colombia, Ministerio de Educación Nacional (2003), "Decreto número 2566 del 10 de septiembre de 2003", [en línea], disponible en: http://www.universia.net. co/universidades/view-document/documento-78. html, recuperado: Abril de 2009.

Colombia, Ministerio de Educación Nacional (2006), “Resolución 2755 de 2006", [en línea], disponible en: http://www.alcaldiabogota.gov.co/sisjur/normas/ Norma1.jsp?i=22447, recuperado: Abril de 2009.

Guzmán, I. (2006, agosto), “La investigación en los nuevos escenarios de la virtualidad”, en Revista Apertura de Innovación Educativa, [en línea], vol. 6, núm. 004, pp. 8-19, México, Universidad de Guadalajara, disponible en: http://redalyc.uaemex.mx/redalyc/src/inicio/ ArtPdfRed.jsp?iCve=68800402, recuperado: Mayo de 2009.

Ligth, D. (2005), REDAL (Redes Escolares de América Latina): Una investigación de las mejores prácticas, [en línea], disponible en http://e-spacio.uned.es/ fez/eserv.php?pid=bibliuned:19404\&dsID=n04lig ht05.pdf, recuperado: Mayo de 2009.

Mauri, T. et al. (2009, enero-abril), “Diseño de propuestas docentes con TIC en la enseñanza superior: nuevos retos y principios de calidad desde una perspectiva socio-constructivista.", en Revista de Educación, [en línea], 348, pp. 377-399, disponible en: http://www. revistaeducacion.mec.es/re348/re348_16.pdf, recuperado: Abril de 2009.
Ocampo G., Rodrigo Jesús (2008, julio-diciembre), “Preliminares para pensar una filosofía de la cibercultura" En Revista El hombre y la máquina, núm.31, pp. 64-77, Cali, Universidad Autónoma de Occidente, Disponible en: http://redalyc.uaemex.mx/src/inicio/ArtPdfRed. jsp?iCve=47803106, recuperado: Agosto de 2009.

Parra, O. (2008, noviembre), "El estudiante adulto en la era digital”, en Revista Apertura, [en línea], vol.8, pp. 35-50, Universidad de Guadalajara, disponible en: http://redalyc.uaemex.mx/src/inicio/ArtPdfRed. jsp?iCve=68811215003, recuperado: Julio de 2009 .

Ramírez, J. (2006, enero-marzo), "Las tecnologías de la información y de la comunicación en la educación en cuatro países latinoamericanos", en Revista Mexicana de Investigación Educativa, [en línea], año/vol., 11, núm. 028, disponible en: http://redalyc.uaemex.mx/ src/inicio/ArtPdfRed.jsp?iCve=14002805, recuperado: Julio de 2009.

Rodríguez Cuberos, E. G. et al. (2007, julio), “Aplicaciones pedagógicas del juego de rol en la educación virtual. Una experiencia en el contexto del Examen de Estado", en Revista Electrónica de Tecnología Educativa, EDUTEC., [en línea], núm. 23, disponible en: http:// edutec.rediris.es/Revelec2/revelec23/ro_lo_cas_di/ ro_lo_cas_di.html, recuperado: Julio de 2009.

Salinas, J. (2004, marzo), "Los entornos tecnológicos en la Universidad", en Pixel-Bit Revista de Medios y Educación, [en línea], núm. 32, pp. 83-101, España, Universidad de las Islas Baleares, disponible en: http://www.sav.us.es/pixelbit/pixelbit/articulos/ n32/6.pdf, recuperado: Mayo de 2009

Sánchez, J. (2006, enero-junio), “Panorama de la investigación sobre políticas de formación en América Latina", en Revista Interamericana de Bibliotecología, [en línea], vol. 29, núm.1, Medellín, Colombia, disponible en: http://www.scielo.org.co/scielo.php?pid=S012009762006000100007\&script=sci_arttext\&tlng=es, Recuperado: Julio de 2009

Torres, A. (2005), "Redes académicas en los entornos virtuales”, en Revista Apertura, [en línea], vol. 5, núm. 001, pp. 83-91, México, Universidad de Guadalajara, disponible en: http://redalyc.uaemex.mx/src/inicio/ArtPdfRed.jsp?iCve=68850109\&iCveNum=8734, recuperado: Mayo de 2009. 